\title{
Efeito da substituição do feno de Tifton 85 pelo caroço de algodão como fonte de fibra na dieta de bezerros
}

\author{
[Effect of substitution of Tifton 85 hay by whole cotton seed as fiber source in the diets of calves] \\ E.B. Bernardes ${ }^{1}$, S.G. Coelho ${ }^{2 *}$, A.U. Carvalho ${ }^{2}$, H.N. Oliveira ${ }^{3}$, R.B. Reis ${ }^{2}$, \\ H.M. Saturnino ${ }^{2}$, C.A. Silva ${ }^{4}$, T.C. Costa ${ }^{1}$ \\ ${ }^{1}$ Médico veterinário autônomo \\ ${ }^{2}$ Escola de Veterinária - UFMG \\ Caixa Postal 567 \\ 30123-970 - Belo Horizonte, MG \\ ${ }^{3}$ Faculdade de Medicina Veterinária e Zootecnia - UNESP - Botucatu, SP \\ ${ }^{4}$ Médica veterinária autônoma
}

\begin{abstract}
RESUMO
Para avaliar a substituição do feno Tifton 85 pelo caroço de algodão como fonte de fibra na dieta foram utilizados 24 bezerros da raça Holandesa, distribuídos em dois grupos. O grupo-feno recebeu feno e concentrado separados, fornecidos à vontade, e o grupo- algodão recebeu caroço de algodão incorporado ao concentrado $(13,5 \%)$, à vontade. O consumo de alimentos, o peso, as alturas da cernelha e do íleo, as circunferências torácica e abdominal, a concentração de glicose sangüínea, as concentrações de acetato, propionato e butirato, o $\mathrm{pH}$ do fluido ruminal e os pesos dos compartimentos do estômago foram mensurados. O consumo de alimentos, o desenvolvimento ponderal dos animais, as concentrações de acetato, propionato e butirato, o $\mathrm{pH}$ do fluido ruminal, as concentrações de glicose sangüíneas e o peso dos compartimentos do estômago foram semelhantes entre os tratamentos $(\mathrm{P}>0,05)$. À necropsia, não foram observadas alterações sugestivas de intoxicação por gossipol. Concluiu-se que o caroço de algodão substituiu, sem prejuízos, o feno como fonte de fibra na dieta de bezerros.
\end{abstract}

Palavras-chave: bezerro, AGV, desempenho, glicose sangüínea, pH ruminal

\begin{abstract}
Twenty-four Holstein calves divided into two groups were used to evaluate Tifton 85 hay or whole cotton seed as fiber source in their diets. One group received Tifton 85 hay and concentrate ad libitum (hay group) and the other received ad libitum (cotton group) only concentrate with whole cotton seed incorporated (13,5\%). Feed intake; weight; whither and ileum heights; thoracic and abdominal circumferences; acetate, propionate and butyric concentrations; $p H$ of ruminal content; blood glucose; and rumen, reticulum, omasum and abomasum weights were measured. Feed intake; animal performance; acetate, propionate and butyric concentrations; $p H$ of ruminal content; blood glucose and weight of stomach compartments did not differ between the groups (P>0.05). No clinical evidence of gossypol intoxication was observed. It was concluded that whole cotton seed replaced hay as fiber source in the diets of calves.
\end{abstract}

Keywords: calf, VFA, performance, blood glucose, rumen $\mathrm{pH}$

Recebido em 6 de outubro de 2006

Aceito em 6 de junho de 2007

*Autor para correspondência (corresponding author)

E-mail: sandra@vet.ufmg.br

Apoio: FAPEMIG 


\section{Bernardes et al.}

\section{INTRODUÇÃO}

A utilização de fontes de fibra na dieta de bezerros continua sendo um assunto controverso e, cada vez mais, maior número de criadores tem optado por não fornecer volumosos para animais até 60 ou 90 dias de idade. A opção pelo não fornecimento de volumosos deve-se ao conhecimento de que o desenvolvimento do epitélio do rúmen está diretamente relacionado ao consumo de alimentos ricos em carboidratos. No entanto, algum alimento que provoque a movimentação ruminal e ruminação é necessário para manutenção da saúde do rúmen retículo (Anderson et al., 1982).

Dietas compostas apenas por alimentos concentrados e com baixo teor de fibra podem provocar queda do $\mathrm{pH}$ ruminal, paraceratose e hiperqueratinização das papilas ruminais, resultando em redução na ingestão de matéria seca e na absorção de ácidos graxos voláteis (AGV) (Nocek et al., 1984; Plaza et al., 1990).

O feno de boa qualidade tem sido o alimento mais recomendado como fonte de fibra a ser fornecida para bezerros até quatro meses de idade. No entanto, ele é considerado o alimento volumoso mais caro por unidade de matéria seca conservada. Além disso, ocorre grande desperdício quando este é oferecido a animais jovens. Dessa forma, apesar de a utilização de feno inteiro, ou picado em grandes partículas, não ser adequada na dieta de bezerros, a adição de fontes de fibra em partículas menores se faz necessária. A incorporação de 10 a $25 \%$ de feno picado, ou outra fonte de fibra de boa qualidade, ao concentrado, aumenta o consumo de matéria seca e o ganho de peso dos animais (Nocek et al., 1984). A forma física da dieta, em particular o tamanho das partículas, influencia o consumo de alimentos, o ganho de peso e a saúde dos bezerros. Sabe-se que o tamanho das partículas é mais importante que o próprio teor de fibra da dieta quando se trata da saúde do rúmen de bezerros até oito semanas de idade.

Com o objetivo de reduzir o custo das dietas de bezerros, faz-se necessária a busca de outras fontes de fibra que devem ser nutritivas e ao mesmo tempo economicamente interessantes. Os subprodutos da agroindústria aparecem como alternativas viáveis, sendo o caroço de algodão um dos mais importantes, por apresentar disponibilidade crescente, para uso em ração animal. Associado a isto, apresenta altas concentrações de energia, de proteína e de fibra permitindo a substituição de alimentos volumosos sem prejudicar a fermentação ruminal (Nutrient..., 1989; Delgado, 1994). Um ponto negativo para sua utilização é o gossipol, um pigmento natural, encontrado no algodão que é tóxico para monogástricos e ruminantes.

O objetivo deste trabalho foi avaliar a substituição do feno pelo caroço de algodão como fonte de fibra na dieta de bezerros observando o seu consumo e os reflexos sobre o desempenho dos animais, o perfil de fermentação, a saúde e o desenvolvimento ruminal, e a concentração sangüínea de glicose, bem como algum efeito deletério sobre a saúde dos animais.

\section{MATERIAL E MÉTODOS}

Foram utilizados 24 bezerros machos da raça Holandesa distribuídos em dois grupos experimentais (12 para cada grupo). Os animais foram identificados, pesados e mensurados (altura da cernelha e ílio, e circunferências torácica e abdominal) e alojados em gaiolas individuais onde permaneceram até os 90 dias de idade.

Os animais receberam quatro litros de colostro no primeiro dia de vida, quatro litros de leite integral do segundo ao sétimo dia e quatro litros de sucedâneo de leite do oitavo ao $30^{\circ}$ dia, quando foram desaleitados. Foi utilizado sucedâneo comercial com os seguintes níveis de garantia mínimos (mín) e máximos (máx): umidade $10,0 \%$ (máx), proteína bruta (PB) 22,0\% (mín), extrato etéreo (EE) 8,0\% (mín), fibra bruta (FB) 3,0\% (máx), matéria mineral (MM) 15\% (máx), cálcio (Ca) 1,5\% (máx), fósforo (F) $0,7 \%$ (mín) e lactose $25 \%$, na diluição 1:14.

Os grupos receberam o mesmo manejo e alimentos concentrados com teores semelhantes de $\mathrm{PB}, \mathrm{EE}$, minerais e relação cálcio: fósforo. $\mathrm{O}$ que diferiu entre os grupos foram as fontes de fibra e os teores de fibra em detergente neutro (FDN) e fibra em detergente ácido (FDA). No grupo-feno, os animais receberam água, concentrado e feno de Tifton 85 picado $(4 \mathrm{~cm}$ de comprimento) fornecidos separadamente, no 
grupo-algodão, água, concentrado e caroço de algodão incorporado ao concentrado. A composição dos concentrados e a composição bromatológica dos ingredientes utilizados, dos concentrados e do feno podem ser observadas nas Tab. 1 e 2, respectivamente.

Tabela 1. Composição dos concentrados fornecidos aos bezerros, expressa em porcentagem da matéria natural

\begin{tabular}{lcc}
\hline \multirow{2}{*}{ Ingrediente } & \multicolumn{2}{c}{ Grupo } \\
\cline { 2 - 3 } & Feno & Algodão \\
\hline Milho & 61,4 & 56,7 \\
Farelo de soja & 21,1 & 25,1 \\
Soja grão tostada* & 12,9 & - \\
Caroço de algodão & - & 13,5 \\
Sal mineral & 3,0 & 2,6 \\
Calcário & 1,7 & 2,0 \\
\hline *No concentrado do grupo-feno adicionou-se soja grão \\
tostada para que o teor de extrato etéreo se aproximasse ao do \\
concentrado do grupo-algodão.
\end{tabular}

O caroço de algodão foi incorporado inteiro ao concentrado do grupo-algodão para a avaliação de sua efetividade como fonte de fibra na dieta.
Concentrado, feno e água foram fornecidos à vontade a partir do $3^{\circ}$ dia e até os 90 dias de idade. $\mathrm{O}$ consumo foi avaliado às sete horas, diariamente, por meio da diferença entre $\mathrm{o}$ fornecido e as sobras.

Amostras dos alimentos fornecidos e de suas sobras foram coletadas para análises. Foram determinadas, segundo AOAC (Official..., 1980), a MS a $105^{\circ} \mathrm{C}$ e a $\mathrm{PB}$ pelo método de Kjeldhal. Os componentes da parede celular (FDN e FDA) foram determinados pelo método seqüencial proposto por Van Soest et al. (1991). Os teores de cinzas e EE foram determinados segundo Silva (1981).

O desempenho dos animais foi avaliado pela pesagem e pela mensuração da altura da cernelha e ílio e circunferências torácica e abdominal (atrás da última costela). Esses procedimentos foram realizados pela manhã, antes do fornecimento dos alimentos e a cada sete dias até o final do experimento.

Tabela 2. Composição bromatológica dos ingredientes utilizados na formulação dos alimentos concentrados, do feno de Tifton 85, dos concentrados fornecidos aos animais, expressa em porcentagem da matéria seca

\begin{tabular}{lcccccccc}
\hline & MS & PB & EE & FDN & FDA & Cinzas & Ca & P \\
\hline Caroço de algodão & 91,0 & 24,3 & 23,9 & 44,2 & 33,8 & 4,07 & 0,2 & 0,6 \\
Soja grão tostada & 92,0 & 38,2 & 23,5 & 22,2 & 15,8 & 4,86 & 0,2 & 0,2 \\
Farelo de soja & 88,0 & 52,0 & 1,8 & 12,5 & 9,3 & 6,56 & 0,3 & 0,6 \\
Milho & 86,0 & 10,0 & 5,8 & 15,2 & 4,1 & 1,40 & 0,1 & 0,3 \\
Sal mineral & 99,0 & - & - & - & - & - & 11,3 & 8,0 \\
Calcário & 99,0 & - & - & - & - & - & 38,3 & - \\
Feno & 87,4 & 14,9 & 2,3 & 79,1 & 42,5 & 7,47 & 0,7 & 0,2 \\
Concentrado do grupo-feno & 90,2 & 21,3 & 5,8 & 12,5 & 7,6 & 6,64 & 1,1 & 0,6 \\
Concentrado do grupo-algodão & 90,8 & 21,6 & 6,2 & 17,8 & 9,4 & 6,53 & 1,2 & 0,6 \\
\hline
\end{tabular}

Seis animais de cada grupo foram sorteados para serem doadores de fluido de rúmen e sangue. O perfil de fermentação ruminal foi avaliado mediante mensuração do $\mathrm{pH}$ e concentrações dos AGVs do fluido ruminal aos 30, 45, 60, 75 e 90 dias de idade. Uma alíquota de fluido ruminal de cada bezerro foi retirada por meio de sonda, como proposto por Ortolani (1981) e modificado por Coelho (1999). As coletas foram realizadas antes do fornecimento do alimento no período da manhã, e três, seis e nove horas após a primeira coleta. A mensuração do $\mathrm{pH}$ foi realizada após a filtragem do fluido ruminal, imediatamente após a colheita, utilizando-se potenciômetro portátil ${ }^{1}$. Para a análise de AGVs nas amostras de fluido ruminal, foi adicionado $1 \mathrm{ml}$ de ácido metafosfórico a $20 \%$ na proporção de uma parte do ácido para cinco da amostra. Para serem analisadas, as amostras foram centrifugadas a 10.000rpm por 25 minutos e filtradas em filtro milipore $0,45 \mu \mathrm{m}$ de poro por $25 \mathrm{~mm}$. Os AGVs foram quantificados usando-se cromatógrafo de

${ }^{1}$ Digimed, modelo DM-2 - São Paulo, Brasil. 
fase gasosa ${ }^{2}$, com coluna de vidro de dois metros de comprimento e diâmetro de $1 / 8$ de polegada com chromosorb 101 (80 - 100mesh), conforme Russel (1988).

A concentração sanguínea de glicose foi avaliada a cada 15 dias a partir dos 45 dias de idade nos mesmos horários de coleta de suco de rúmen. Foi utilizado aparelho portátil com faixa de medição entre 10 e $600 \mathrm{mg} / \mathrm{dl}^{3}$.

Aos 30, 60 e 90 dias de idade, três bezerros de cada grupo (total de 18 bezerros) foram sacrificados por eletrocussão, precedida de anestesia geral, para avaliação do desenvolvimento dos pré-estômagos e abomaso. Os pré-estômagos e abomaso foram retirados da carcaça, pesados juntos e cheios e, posteriormente, separados em retículo-rúmen, omaso e abomaso e pesados cheios e vazios. Nesses animais foram realizadas necropsias completas para avaliações de possíveis alterações causadas por intoxicação por gossipol.

$\mathrm{O}$ experimento foi montado seguindo $\mathrm{o}$ delineamento inteiramente ao acaso. Os dados foram analisados utilizando-se os procedimentos GLM do software SAS (User's..., 1995). Para os dados de consumo de alimentos sólidos, de água e desempenho dos animais utilizou-se o arranjo em parcelas subdivididas, com os tratamentos nas parcelas e as semanas nas subparcelas. Os dados de AGVs, pH do suco ruminal e glicose sanguínea foram analisados segundo o arranjo em parcelas subsubdivididas, com os tratamentos nas parcelas, as idades de coleta nas subparcelas e os horários de coleta nas subsubparcelas. O desenvolvimento dos pré-estômagos e abomaso foram avaliados por arranjo fatorial: 2 tratamentos $\times 3$ idades de abate (três repetições). A diferença mínima significativa foi calculada por meio do erro de sua respectiva fonte de variação; para testar as diferenças entre as médias usou-se o teste Tukey $(\mathrm{P}<0,05)$.

\section{RESULTADOS E DISCUSSÃO}

Os dados referentes ao consumo médio diário da matéria seca do concentrado e do feno, matéria seca total, água e ganho de peso médio diário dos animais são apresentados na Tab. 3. Não houve diferença $(\mathrm{P}>0,05)$ entre os grupos para essas variáveis. Observou-se que alguns animais do

${ }^{2}$ Varian, modelo 2485 - São Paulo, Brasil.

${ }^{3}$ Roche Diagnostics, modelo Advantage - Indianápolis, EUA. grupo-algodão, nas duas primeiras semanas de idade, separavam parte do caroço de algodão e não o consumiam. Entretanto, essa seleção parcial não refletiu em diferença $(\mathrm{P}>0,05)$ entre as concentrações de nutrientes do concentrado fornecido e suas sobras. Não foram observados caroços de algodão inteiros nas fezes dos animais ou no conteúdo do abomaso dos animais sacrificados aos 30 dias de idade, demonstrando que os animais foram capazes de digerir o caroço em idade precoce, como relatado por Anderson et al. (1982).

O consumo médio diário de matéria seca dos alimentos concentrados e de matéria seca total bem como o ganho de peso médio diário foram diferentes entre as semanas estudadas $(\mathrm{P}<0,05)$. $\mathrm{O}$ consumo de matéria seca total manteve-se baixo até o desaleitamento, representando para os animais do grupo-feno $1,5 \%$ e $1,2 \%$ do peso vivo (PV) para o grupo-algodão na quarta semana de idade. Na quinta e sexta semanas o consumo aumentou, em média, 69,3\% em relação ao da quarta semana, atingindo valores médios de 2,4 e 2,3\% para os dois grupos, respectivamente. Este aumento foi causado pelo desaleitamento que forçou os animais a aumentar a ingestão de alimentos para suprir suas exigências nutricionais. A partir da oitava semana o consumo praticamente se estabilizou em 2,9\% do PV nos animais dos dois grupos.

O consumo médio diário de matéria seca de feno mostrou-se baixo e variável durante todo o experimento. Resultados semelhantes também foram observados por Plaza et al. (1990), Coelho (1999) e Fontes et al. (2006) ao relatarem que os bezerros consomem mais concentrado e menos feno quando o feno e o concentrado são fornecidos separadamente. Isso se deve, provavelmente, à maior palatabilidade do concentrado, à dificuldade dos animais em apreender o feno e à sua menor degradabilidade.

A variação no consumo de feno ocorre principalmente após grande ingestão de concentrado e pode ser uma tentativa do animal em regular o $\mathrm{pH}$ ruminal mediante consumo de alimentos que proporcionam taxa de degradação mais lenta com menor queda do $\mathrm{pH}$ ruminal após a alimentação, como relatou Coelho (1999). 
Tabela 3. Consumo médio diário de matéria seca do concentrado (conc) e do feno, de matéria seca total (MST), em gramas, de água, em litros, e ganho médio de peso diário (GPD), em gramas, de bezerros alimentados com concentrado e feno (grupo-feno) ou com concentrado acrescido de caroço de algodão (grupo-algodão), segundo a idade, em semanas

\begin{tabular}{|c|c|c|c|c|c|c|c|c|c|}
\hline \multirow{2}{*}{$\begin{array}{l}\text { Idade } \\
\text { (semanas) }\end{array}$} & \multicolumn{5}{|c|}{ Grupo-feno } & \multicolumn{4}{|c|}{ Grupo-algodão } \\
\hline & Conc $^{1}$ & Feno & $\mathrm{MST}^{2}$ & Água $^{3}$ & $\mathrm{GPD}^{4}$ & Conc & MST & Água & GPD \\
\hline 1 & $53,6 \mathrm{I}$ & 0 & $53,6 \mathrm{I}$ & $1,1 \mathrm{H}$ & $242,0 \mathrm{C}$ & $42,7 \mathrm{H}$ & $42,7 \mathrm{H}$ & $1,5 \mathrm{~F}$ & $291,0 \mathrm{C}$ \\
\hline 2 & $171,4 \mathrm{I}$ & 3,0 & $174,4 \mathrm{I}$ & $1,3 \mathrm{H}$ & $-30,0 \mathrm{D}$ & $82,6 \mathrm{H}$ & $82,6 \mathrm{H}$ & $1,5 \mathrm{~F}$ & $-54,0 \mathrm{D}$ \\
\hline 3 & $226,1 \mathrm{I}$ & 17,0 & $243,1 \mathrm{I}$ & $0,9 \mathrm{H}$ & $-8,0 \mathrm{D}$ & $171,1 \mathrm{H}$ & $171,1 \mathrm{H}$ & $1,1 \mathrm{~F}$ & $-67,0 \mathrm{D}$ \\
\hline 4 & $591,6 \mathrm{H}$ & 6,5 & $598,1 \mathrm{H}$ & $1,5 \mathrm{H}$ & $606,0 \mathrm{BC}$ & $450,3 \mathrm{G}$ & $450,3 \mathrm{G}$ & $1,3 \mathrm{~F}$ & $447,0 \mathrm{BC}$ \\
\hline 5 & $943,1 \mathrm{G}$ & 57,0 & $1000,1 \mathrm{FG}$ & $3,1 \mathrm{FG}$ & $782,0 \mathrm{~B}$ & $825,1 \mathrm{FG}$ & $825,1 \mathrm{FG}$ & $2,9 \mathrm{~F}$ & $596,0 \mathrm{BC}$ \\
\hline 6 & $1181,5 \mathrm{FG}$ & 70,4 & $1251,9 \mathrm{DEF}$ & $3,9 \mathrm{EF}$ & $564,0 \mathrm{BC}$ & $1091,3 \mathrm{EF}$ & $1091,3 \mathrm{EF}$ & $3,8 \mathrm{E}$ & $722,0 \mathrm{BC}$ \\
\hline 7 & $1413,3 \mathrm{EF}$ & 76,4 & $1489,7 \mathrm{CDE}$ & $5,0 \mathrm{DE}$ & $640,0 \mathrm{BC}$ & $1356,1 \mathrm{DE}$ & $1356,1 \mathrm{DE}$ & $4,8 \mathrm{DE}$ & $658,0 \mathrm{BC}$ \\
\hline 8 & $1564,5 \mathrm{E}$ & 110,8 & $1675,3 \mathrm{CD}$ & $5,5 \mathrm{CD}$ & $808,0 \mathrm{~B}$ & $1538,9 \mathrm{CD}$ & $1538,9 \mathrm{CD}$ & $5,2 \mathrm{CDE}$ & $897,0 \mathrm{AB}$ \\
\hline 9 & $1684,5 \mathrm{DE}$ & 109,1 & $1793,7 \mathrm{C}$ & $5,8 \mathrm{CD}$ & $994,0 \mathrm{BC}$ & $1770,0 \mathrm{BC}$ & $1770,0 \mathrm{BC}$ & $5,8 \mathrm{CD}$ & $851,0 \mathrm{~B}$ \\
\hline 10 & $1846,4 \mathrm{CD}$ & 153,3 & $1999,7 \mathrm{BC}$ & $6,9 \mathrm{BC}$ & $873,0 \mathrm{~B}$ & $1920,3 \mathrm{BC}$ & $1920,3 \mathrm{BC}$ & $6,6 \mathrm{BC}$ & $815 \mathrm{~B}, 0 \mathrm{C}$ \\
\hline 11 & $2200,1 \mathrm{C}$ & 136,4 & $2336,5 \mathrm{AB}$ & $8,7 \mathrm{AB}$ & $1057,0 \mathrm{AB}$ & $2028,3 \mathrm{AB}$ & $2028,3 \mathrm{AB}$ & $8,2 \mathrm{AB}$ & $901,0 \mathrm{AB}$ \\
\hline 12 & $2478,7 \mathrm{AB}$ & 100,6 & $2579,3 \mathrm{~A}$ & $8,8 \mathrm{~A}$ & $876,0 \mathrm{~B}$ & $2105,7 \mathrm{AB}$ & $2105,7 \mathrm{AB}$ & $8,7 \mathrm{~A}$ & $629,0 \mathrm{BC}$ \\
\hline 13 & $2741,8 \mathrm{~A}$ & 126,7 & $2868,5 \mathrm{~A}$ & $9,5 \mathrm{~A}$ & $1551,0 \mathrm{~A}$ & $2349,0 \mathrm{~A}$ & $2349,0 \mathrm{~A}$ & $9,1 \mathrm{~A}$ & $1472,0 \mathrm{~A}$ \\
\hline
\end{tabular}

Médias seguidas de letras distintas na mesma coluna diferem entre si $(\mathrm{P}<0,05)$

CV Conc: 20,3\%; CV MST: 23,3\%; CV água: 22,1\%; CV GPD: 51,1\%.

O ganho médio de peso diário (GPD) (Tab. 3) também foi baixo durante o aleitamento devido a quadros de diarréia que acometeram todos os animais na segunda e terceira semanas de idade. Essas diarréias podem ter sido causadas pelo grande desafio imunológico a que estão submetidos os animais nessa idade. Após o desaleitamento o GPD foi elevado devido à recuperação dos animais e ao aumento no consumo de alimentos.

O consumo de água não diferiu entre os tratamentos $(\mathrm{P}>0,05)$. A correlação entre $\mathrm{O}$ consumo de água e matéria seca total foi de 0,93 .

Os dados referentes ao peso, alturas de cernelha e íleo e circunferências torácica e abdominal são apresentados na Tab. 4. Não houve diferença $(\mathrm{P}>0,05)$ para essas características entre os grupos estudados.
As diarréias registradas durante o primeiro mês de vida não influenciaram o crescimento em altura e as circunferências torácica e abdominal. Esse fato está de acordo com as observações de Virtala et al. (1996), segundo os quais o desenvolvimento em altura não é afetado pelas doenças mais comuns que ocorrem em bezerros durante a fase inicial da vida.

$\mathrm{Na}$ Tab. 5 observam-se os pesos dos préestômagos e abomaso. Os pesos dos compartimentos do estômago não diferiram entre os grupos $(\mathrm{P}>0,05)$. Quando se associa essa observação à ausência de diferença de consumo de concentrado e matéria seca total entre os dois grupos (Tab. 3), conclui-se que a dieta com caroço de algodão, mesmo tendo tamanho de partícula inferior, foi capaz de estimular a movimentação e o desenvolvimento dos préestômagos tanto quanto a dieta com feno. 


\section{Bernardes et al.}

Tabela 4. Peso, alturas de cernelha e de íleo (AC e AI) e circunferências torácica e abdominal (CT e CA) de bezerros alimentados com feno e concentrado (grupo-feno) ou concentrado com caroço de algodão (grupo-algodão) do nascimento até a $13^{\mathrm{a}}$ semana de idade

\begin{tabular}{ccccccccccc}
\hline & \multicolumn{2}{c}{ Peso (kg) } & \multicolumn{2}{c}{$\mathrm{AC}(\mathrm{cm})$} & \multicolumn{2}{c}{$\mathrm{AI}(\mathrm{cm})$} & \multicolumn{2}{c}{$\mathrm{CT}(\mathrm{cm})$} & \multicolumn{2}{c}{ CA (cm) } \\
\cline { 2 - 12 } Semana & $\begin{array}{c}\text { Grupo } \\
\text { feno }\end{array}$ & $\begin{array}{c}\text { Grupo } \\
\text { algodão }\end{array}$ & $\begin{array}{c}\text { Grupo } \\
\text { feno }\end{array}$ & $\begin{array}{c}\text { Grupo } \\
\text { algodão }\end{array}$ & $\begin{array}{c}\text { Grupo } \\
\text { feno }\end{array}$ & $\begin{array}{c}\text { Grupo } \\
\text { algodão }\end{array}$ & $\begin{array}{c}\text { Grupo } \\
\text { feno }\end{array}$ & $\begin{array}{c}\text { Grupo } \\
\text { algodão }\end{array}$ & $\begin{array}{c}\text { Grupo } \\
\text { feno }\end{array}$ & $\begin{array}{c}\text { Grupo } \\
\text { algodão }\end{array}$ \\
\hline Nasc. & $34,1 \mathrm{~J}$ & $35,5 \mathrm{~J}$ & $72,7 \mathrm{~L}$ & $74,1 \mathrm{~J}$ & $75,8 \mathrm{~K}$ & $76,1 \mathrm{~K}$ & $75,0 \mathrm{~L}$ & $76,7 \mathrm{~J}$ & $72,1 \mathrm{~K}$ & $71,7 \mathrm{I}$ \\
1 & $35,6 \mathrm{IJ}$ & $37,3 \mathrm{~J}$ & $73,1 \mathrm{KL}$ & $74,6 \mathrm{~J}$ & $76,2 \mathrm{~K}$ & $77,0 \mathrm{JK}$ & $77,7 \mathrm{KL}$ & $77,4 \mathrm{~J}$ & $75,2 \mathrm{~K}$ & $72,9 \mathrm{I}$ \\
2 & $35,4 \mathrm{IJ}$ & $36,9 \mathrm{~J}$ & $74,0 \mathrm{KL}$ & $75,8 \mathrm{IJ}$ & $76,5 \mathrm{~K}$ & $77,9 \mathrm{IJK}$ & $78,6 \mathrm{JK}$ & $78,7 \mathrm{IJ}$ & $75,9 \mathrm{JK}$ & $74,5 \mathrm{HI}$ \\
3 & $35,4 \mathrm{IJ}$ & $36,4 \mathrm{~J}$ & $74,6 \mathrm{JK}$ & $76,2 \mathrm{HI}$ & $77,3 \mathrm{JK}$ & $78,2 \mathrm{IJ}$ & $79,7 \mathrm{JK}$ & $80,8 \mathrm{HI}$ & $77,7 \mathrm{IJ}$ & $76,6 \mathrm{HI}$ \\
4 & $39,6 \mathrm{I}$ & $39,6 \mathrm{IJ}$ & $75,6 \mathrm{IJ}$ & $77,2 \mathrm{GHI}$ & $78,2 \mathrm{IJ}$ & $79,3 \mathrm{GHI}$ & $81,3 \mathrm{IJ}$ & $81,8 \mathrm{HI}$ & $81,5 \mathrm{I}$ & $79,9 \mathrm{GH}$ \\
5 & $45,5 \mathrm{GH}$ & $43,7 \mathrm{HI}$ & $77,0 \mathrm{HI}$ & $78,0 \mathrm{GH}$ & $79,3 \mathrm{HI}$ & $80,4 \mathrm{FGH}$ & $84,1 \mathrm{HI}$ & $83,7 \mathrm{GH}$ & $86,3 \mathrm{HI}$ & $84,6 \mathrm{FG}$ \\
6 & $50,1 \mathrm{FG}$ & $48,8 \mathrm{GH}$ & $78,7 \mathrm{GH}$ & $79,2 \mathrm{FG}$ & $80,7 \mathrm{GH}$ & $81,0 \mathrm{EFG}$ & $86,0 \mathrm{GH}$ & $85,4 \mathrm{FG}$ & $89,9 \mathrm{GH}$ & $88,2 \mathrm{~F}$ \\
7 & $54,8 \mathrm{~F}$ & $53,3 \mathrm{G}$ & $79,8 \mathrm{FG}$ & $80,3 \mathrm{EF}$ & $82,2 \mathrm{FG}$ & $82,4 \mathrm{EF}$ & $88,3 \mathrm{FG}$ & $88,4 \mathrm{EF}$ & $92,3 \mathrm{FG}$ & $90,6 \mathrm{EF}$ \\
8 & $60,7 \mathrm{E}$ & $59,7 \mathrm{EF}$ & $81,2 \mathrm{EF}$ & $82,1 \mathrm{DE}$ & $83,2 \mathrm{EF}$ & $83,0 \mathrm{DE}$ & $91,2 \mathrm{EF}$ & $91,3 \mathrm{DE}$ & $96,1 \mathrm{EF}$ & $96,0 \mathrm{DE}$ \\
9 & $64,4 \mathrm{E}$ & $64,5 \mathrm{DE}$ & $82,3 \mathrm{DE}$ & $82,9 \mathrm{D}$ & $84,6 \mathrm{DE}$ & $85,1 \mathrm{CD}$ & $94,3 \mathrm{DE}$ & $94,0 \mathrm{CD}$ & $101,7 \mathrm{DE}$ & $101,0 \mathrm{CD}$ \\
10 & $70,6 \mathrm{D}$ & $69,1 \mathrm{CD}$ & $83,6 \mathrm{CD}$ & $84,4 \mathrm{CD}$ & $85,8 \mathrm{CD}$ & $86,2 \mathrm{C}$ & $95,9 \mathrm{CD}$ & $95,6 \mathrm{CD}$ & $106,7 \mathrm{CD}$ & $107,4 \mathrm{BC}$ \\
11 & $77,9 \mathrm{BC}$ & $75,1 \mathrm{BC}$ & $84,9 \mathrm{BC}$ & $85,7 \mathrm{BC}$ & $87,0 \mathrm{BC}$ & $87,4 \mathrm{BC}$ & $99,2 \mathrm{C}$ & $98,4 \mathrm{BC}$ & $111,9 \mathrm{BC}$ & $110,0 \mathrm{~B}$ \\
12 & $84,1 \mathrm{~B}$ & $79,2 \mathrm{AB}$ & $87,1 \mathrm{AB}$ & $88,3 \mathrm{AB}$ & $88,2 \mathrm{AB}$ & $89,4 \mathrm{AB}$ & $104,0 \mathrm{~B}$ & $102,8 \mathrm{AB}$ & $115,5 \mathrm{~B}$ & $112,4 \mathrm{AB}$ \\
13 & $91,7 \mathrm{~A}$ & $86,0 \mathrm{~A}$ & $88,4 \mathrm{~A}$ & $89,8 \mathrm{~A}$ & $89,8 \mathrm{~A}$ & $90,8 \mathrm{~A}$ & $107,4 \mathrm{~A}$ & $103,8 \mathrm{~A}$ & $123,2 \mathrm{~A}$ & $119,0 \mathrm{~A}$ \\
\hline
\end{tabular}

Médias seguidas de letras distintas na mesma coluna diferem entre si $(\mathrm{P}<0,05)$.

CVpeso: 5,9\%; CVAC: 1,5\%; CVAI: $1,3 \%$; CVCT: $2,2 \%$; CVCA: $3,9 \%$.

Tabela 5. Peso dos pré-estômagos e abomaso, em quilogramas, cheios e vazios, aos 30, 60 e 90 dias de idade, de bezerros alimentados com feno e concentrado (grupo-feno) ou concentrado com caroço de algodão (grupo-algodão)

\begin{tabular}{|c|c|c|c|c|c|c|c|c|c|c|}
\hline \multirow{3}{*}{$\begin{array}{l}\text { Idade } \\
\text { (dias) }\end{array}$} & \multirow{2}{*}{\multicolumn{2}{|c|}{ Peso ao abate }} & \multirow{2}{*}{\multicolumn{2}{|c|}{ Total }} & \multicolumn{6}{|c|}{ Peso dos compartimentos do estômago cheios (kg) } \\
\hline & & & & & \multicolumn{2}{|c|}{ Retículo-rúmen } & \multicolumn{2}{|c|}{ Omaso } & \multicolumn{2}{|c|}{ Abomaso } \\
\hline & $\begin{array}{c}\text { Grupo } \\
\text { feno }\end{array}$ & $\begin{array}{l}\text { Grupo } \\
\text { algodão }\end{array}$ & $\begin{array}{c}\text { Grupo } \\
\text { feno }\end{array}$ & $\begin{array}{c}\text { Grupo } \\
\text { algodão }\end{array}$ & $\begin{array}{l}\text { Grupo } \\
\text { feno }\end{array}$ & $\begin{array}{c}\text { Grupo } \\
\text { algodão }\end{array}$ & $\begin{array}{c}\text { Grupo } \\
\text { feno }\end{array}$ & $\begin{array}{c}\text { Grupo } \\
\text { algodão }\end{array}$ & $\begin{array}{c}\text { Grupo } \\
\text { feno }\end{array}$ & $\begin{array}{l}\text { Grupo } \\
\text { algodão }\end{array}$ \\
\hline 30 & $33,6 \mathrm{C}$ & $39,7 \mathrm{C}$ & $4,0 \mathrm{~B}$ & $3,6 \mathrm{~B}$ & $2,9 \mathrm{~B}$ & $2,2 \mathrm{~B}$ & $0,2 \mathrm{~B}$ & $0,2 \mathrm{~B}$ & $0,9 \mathrm{~B}$ & $1,0 \mathrm{~B}$ \\
\hline 60 & $68,8 \mathrm{~B}$ & $64,4 \mathrm{~B}$ & $8,6 \mathrm{~A}$ & $8,9 \mathrm{~A}$ & $6,8 \mathrm{~A}$ & $6,5 \mathrm{~A}$ & $0,7 \mathrm{~A}$ & $0,7 \mathrm{~A}$ & $1,1 \mathrm{~B}$ & $1,5 \mathrm{~B}$ \\
\hline 90 & $90,5 \mathrm{~A}$ & $89,6 \mathrm{~A}$ & $11,0 \mathrm{~A}$ & $11,4 \mathrm{~A}$ & $8,6 \mathrm{~A}$ & $9,3 \mathrm{~A}$ & $1,1 \mathrm{~A}$ & $0,9 \mathrm{~A}$ & $1,5 \mathrm{~A}$ & $1,2 \mathrm{~A}$ \\
\hline & & & & & \multicolumn{6}{|c|}{ Peso dos compartimentos do estômago vazios (kg) } \\
\hline 30 & $33,6 \mathrm{C}$ & $39,7 \mathrm{C}$ & $0,8 \mathrm{~B}$ & $0,9 \mathrm{~B}$ & $0,5 \mathrm{~B}$ & $0,5 \mathrm{~B}$ & $0,1 \mathrm{~B}$ & $0,1 \mathrm{~B}$ & $0,3 \mathrm{~B}$ & $0,3 \mathrm{~B}$ \\
\hline 60 & $68,8 \mathrm{~B}$ & $64,4 \mathrm{~B}$ & $2,0 \mathrm{~A}$ & $2,0 \mathrm{~A}$ & $1,2 \mathrm{~A}$ & $1,2 \mathrm{~A}$ & $0,4 \mathrm{~A}$ & $0,4 \mathrm{~A}$ & $0,4 \mathrm{~B}$ & $0,4 \mathrm{~B}$ \\
\hline 90 & $90,5 \mathrm{~A}$ & $89,6 \mathrm{~A}$ & $2,7 \mathrm{~A}$ & $2,7 \mathrm{~A}$ & $1,5 \mathrm{~A}$ & $1,7^{\mathrm{a}}$ & $0,8 \mathrm{~A}$ & $0,6 \mathrm{~A}$ & $0,4 \mathrm{~A}$ & $0,4 \mathrm{~A}$ \\
\hline
\end{tabular}

CVpeso ao abate: 14,7\%; CVtotal cheio: 18,8\%; CVretículo - rúmen cheios: 22,6\%; CVomaso cheio: 37,3\%; CVabomaso cheio: 17,1\%; CVtotal vazio: $19,1 \%$; CVretículo-rúmen vazios: $17,4 \%$; CVomaso vazio: $40,0 \%$; CVabomaso vazio: $17,5 \%$.

O aumento no peso dos compartimentos ocorreu em taxas diferentes nas distintas idades. Entre 30 a 60 dias houve mudança acentuada na porcentagem dos pesos do retículo-rúmen e abomaso em relação ao peso total do estômago. Aos 30 dias de idade as proporções do retículorúmen, do omaso e do abomaso foram de 53, 14 e $33 \%$, respectivamente, em relação ao peso total dos pré-estômagos e abomaso. Aos 60 dias, essas proporções foram, respectivamente, de $65,5,15,0$ e $19,5 \%$, enquanto que aos 90 dias, foram de
68,6, 15,7 e 15,7\%. Após os 60 dias, essas proporções praticamente permaneceram nos valores próximos aos relatados por Church (1993) para animais adultos. Essas alterações estão relacionadas ao aumento do consumo de alimentos.

Os valores de $\mathrm{pH}$ do fluido ruminal dos bezerros dos grupos-feno e algodão nas diferentes idades e horários estudados são apresentados na Tab. 6. Não houve diferença $(\mathrm{P}>0,05)$ quanto aos valores 
de $\mathrm{pH}$ do fluido ruminal entre os tratamentos. Foram observadas diferenças $(\mathrm{P}<0,05)$ entre as semanas e entre os horários estudados. Ocorreu queda do $\mathrm{pH}$ do fluido ruminal entre zero e três horas após o fornecimento dos alimentos em função do maior consumo de concentrado, assim que alimentos frescos eram fornecidos aos animais. Essa queda foi seguida quase sempre pelo aumento nos valores às seis e às nove horas.

Tabela 6. $\mathrm{pH}$ do fluido ruminal de bezerros desaleitados aos 30 dias de idade e alimentados com feno e concentrado (grupo-feno) ou concentrado com caroço de algodão (grupo-algodão), imediatamente antes do fornecimento da alimentação (hora zero), três, seis e nove horas após a primeira alimentação do dia

\begin{tabular}{cccccc}
\hline \multirow{2}{*}{$\begin{array}{c}\text { Idade } \\
\text { (dias) }\end{array}$} & Grupo & 0 & 3 & 6 & 9 \\
\cline { 3 - 5 } 30 & Feno & $5,4 \mathrm{Ca}$ & $5,3 \mathrm{Ba}$ & $5,5 \mathrm{Ba}$ & $5,4 \mathrm{Ca}$ \\
& Algodão & $5,8 \mathrm{Ca}$ & $5,4 \mathrm{ABa}$ & $5,4 \mathrm{Ba}$ & $5,6 \mathrm{Ca}$ \\
45 & Feno & $6,1 \mathrm{Ca}$ & $5,7 \mathrm{ABa}$ & $5,6 \mathrm{ABa}$ & $5,4 \mathrm{Ca}$ \\
& Algodão & $5,9 \mathrm{Ca}$ & $5,6 \mathrm{ABa}$ & $5,8 \mathrm{ABa}$ & $5,8 \mathrm{BCa}$ \\
60 & Feno & $6,3 \mathrm{BCa}$ & $6,2 \mathrm{BCa}$ & $5,9 \mathrm{ABa}$ & $6,0 \mathrm{BCa}$ \\
& Algodão & $6,0 \mathrm{Ca}$ & $5,6 \mathrm{Ca}$ & $5,8 \mathrm{ABa}$ & $5,6 \mathrm{Ca}$ \\
75 & Feno & $7,0 \mathrm{ABa}$ & $6,0 \mathrm{ABa}$ & $6,1 \mathrm{ABb}$ & $5,9 \mathrm{BCb}$ \\
& Algodão & $6,8 \mathrm{ABa}$ & $5,8 \mathrm{ABa}$ & $5,8 \mathrm{ABb}$ & $5,9 \mathrm{BCb}$ \\
& Feno & $7,2 \mathrm{Aa}$ & $6,2 \mathrm{Ab}$ & $6,3 \mathrm{Ab}$ & $6,9 \mathrm{Aab}$ \\
& Algodão & $6,9 \mathrm{ABa}$ & $6,1 \mathrm{Ab}$ & $6,3 \mathrm{Ab}$ & $6,5 \mathrm{ABab}$ \\
\hline
\end{tabular}

Médias seguidas de letras maiúsculas distintas na coluna ou letras minúsculas distintas na linha diferem entre si (P<0,05). $\mathrm{CV}: 5,3 \%$.

$\mathrm{O} \mathrm{pH}$ do fluido ruminal dos bezerros de ambos os grupos aos 30 dias de idade se mostrou baixo. Nessa idade, a ruminação ainda é incipiente e o baixo fluxo de tamponantes salivares para o rúmen, associado ao aumento no consumo de concentrado observado na quarta semana de vida dos animais (quase o dobro em relação à semana anterior) justifica a observação destes baixos valores. Segundo Anderson et al. (1982), Vazquéz-Anon (1993) e Beharka et al. (1998), o pH é baixo à época do desaleitamento, pois ocorre o desbalanço entre a produção e a absorção dos AGV já que o rúmen e principalmente o seu epitélio (sítio de metabolismo e absorção de $\mathrm{AGV}$ ) ainda não se encontram plenamente desenvolvidos e a microbiota ruminal característica não está completamente estabelecida, prevalecendo bactérias aminolíticas. Uma outra hipótese a ser considerada seria a baixa qualidade do sucedâneo fornecido aos animais, que pode ter gerado falha no reflexo da goteira esofágica causando desvio do sucedâneo para o rúmen, provocando fermentação intensa e abaixamento do $\mathrm{pH}$.

Entre 45 e 60 dias de idade, o pH médio do fluido ruminal dos bezerros dos grupos-feno e algodão permaneceu baixo e ocorreram três casos clínicos de acidose ruminal (um no grupo- feno e dois no grupo-algodão). Os animais apresentaram timpanismo, diarréia e queda no consumo de alimento concentrado. $\mathrm{O}$ animal do grupo-feno apresentou ainda formação de sola dupla nos quatro cascos. Estes três animais foram sacrificados aos 60 dias de idade, quando então foram observadas áreas de cicatrização nos pilares cranial ou transversal do rúmen e áreas de aglomeração de papilas ou sem papilas (áreas de cicatrização) no saco ventral. Esses achados são semelhantes aos observados por Coelho (1999) que concluiu que bezerros de cerca de 60 dias de idade são propensos a quadros de acidose ruminal, ruminite e paraceratose devido ao elevado consumo de concentrado e à preferência por este em detrimento ao feno.

A partir dos 75 dias de idade, o pH do fluido ruminal dos bezerros de ambos os grupos já se mostrou mais elevado. Mesmo com o aumento de consumo de concentrado e a maior concentração de AGV observados, a queda de $\mathrm{pH}$ três horas após a alimentação foi menor. Isto demonstra melhor capacidade de tamponamento proveniente da ruminação e balanço mais equilibrado entre a quantidade produzida $\mathrm{e}$ absorvida de $\mathrm{AGV}$, de modo que o $\mathrm{pH}$ ruminal se mantém mais elevado (Church, 1993). Aos 90 dias de idade, o pH ruminal dos animais dos dois 
grupos é semelhante ao de um animal adulto e mostra que o caroço de algodão foi tão capaz quanto o feno em manter o $\mathrm{pH}$ ruminal numa faixa favorável ao crescimento de bactérias celulolíticas.

Não foram observadas diferenças $(P>0,05)$ nas concentrações ruminais de AGV entre os tratamentos, idades e horários de coleta para quase todas as variáveis avaliadas. A concentração média total dos AGV aumentou numericamente com o avançar da idade dos animais (Tab. 7), como observado por Anderson et al. (1982) e Quigley et al. (1991) e se estabilizou a partir dos 75 dias de idade. O aumento da concentração total dos AGV acompanha o aumento no consumo de matéria seca total por quilo de peso vivo dos animais. $\mathrm{O}$ aumento da taxa de fermentação ruminal acontece à medida que maior quantidade de substrato (alimento) chega ao rúmen e que a microbiota característica coloniza o órgão (Bergman, 1990; France e Siddons, 1996).

Tabela 7. Concentração média $(\mathrm{mmol} / 100 \mathrm{ml})$ de acetato $(\mathrm{Ac})$, propionato $(\mathrm{Pr})$, butirato $(\mathrm{Bu})$ e de $\mathrm{AGV}$ totais (total), relação acetato: propionato e $\mathrm{pH}$ médio do suco ruminal de bezerros alimentados com feno e concentrado (F) ou concentrado com caroço de algodão (A), de acordo com a idade em dias

\begin{tabular}{|c|c|c|c|c|c|c|c|c|c|c|c|c|}
\hline \multirow{3}{*}{$\begin{array}{l}\text { Idade } \\
\text { (dias) }\end{array}$} & \multicolumn{8}{|c|}{ Concentração média } & \multirow{2}{*}{\multicolumn{2}{|c|}{ Ac: $\operatorname{Pr}$}} & \multirow{2}{*}{\multicolumn{2}{|c|}{$\mathrm{pH}$}} \\
\hline & \multicolumn{2}{|c|}{ Ac } & \multicolumn{2}{|c|}{$\operatorname{Pr}$} & \multicolumn{2}{|c|}{$\mathrm{Bu}$} & \multicolumn{2}{|c|}{ Total } & & & & \\
\hline & $\mathrm{F}$ & A & $\mathrm{F}$ & $\mathrm{A}$ & $\bar{F}$ & A & $\mathrm{F}$ & A & $\mathrm{F}$ & A & $\mathrm{F}$ & A \\
\hline 30 & 6,7 & 7,9 & 6,7 & 9,8 & 0,6 & 0,6 & 14,0 & 18,3 & 1,1 & 0,8 & $5,5 \mathrm{D}$ & $5,6 \mathrm{C}$ \\
\hline 45 & 7,6 & 8,5 & 8,3 & 10,4 & 1,0 & 0,6 & 16,8 & 19,5 & 0,9 & 0,7 & $5,7 \mathrm{CD}$ & $5,8 \mathrm{BC}$ \\
\hline 60 & 8,0 & 8,5 & 8,3 & 9,4 & 0,8 & 0,6 & 17,1 & 18,6 & 1,0 & 0,9 & $6,1 \mathrm{BC}$ & $5,7 \mathrm{BC}$ \\
\hline 75 & 8,5 & 10,4 & 7,8 & 10,8 & 1,6 & 1,3 & 18,0 & 22,5 & 1,3 & 1,0 & $6,2 \mathrm{AB}$ & $6,1 \mathrm{AB}$ \\
\hline 90 & 8,0 & 10,0 & 6,5 & 9,0 & 3,6 & 1,3 & 18,1 & 20,3 & 1,3 & 1,2 & $6,6 \mathrm{~A}$ & $6,5^{\mathrm{a}}$ \\
\hline
\end{tabular}

Médias seguidas de letras distintas na mesma coluna diferem entre si $(\mathrm{P}<0,05)$.

CVAC: 34,0\%; CVPr:=33,8\%; CVBu: 158,0\%; CVtotal: 34,4\%; CVAc:Pr: 18,1\%; CVpH: 5,3\%.

A relação acetato:propionato aumenta com a idade, provavelmente em função da maior capacidade de absorção do rúmen causada por aumento de tamanho do órgão (Tab. 5) e tamponamento do ambiente ruminal nos dois grupos. Aos 90 dias de idade essa relação e os valores de $\mathrm{pH}$ do conteúdo ruminal são semelhantes nos dois grupos, indicando, mais uma vez, a capacidade do caroço em manter bom ambiente ruminal.

As concentrações sangüíneas de glicose dos animais dos grupos-feno e algodão nas diferentes idades e horários de coleta são observadas na Tab. 8. Não foram observadas diferenças $(\mathrm{P}>0,05)$ entre os tratamentos, idades ou horários de coleta, causadas pela ausência de diferenças entre o consumo de alimentos concentrados e matéria seca total, e a concentração média de propionato entre os grupos. A queda numérica dos valores de glicose com o avançar da idade é um reflexo do desenvolvimento ruminal e da adaptação metabólica dos animais que substituem lactose e lactato pelo propionato como principal precursor da síntese de glicose no fígado à medida que passam da condição de préruminante para ruminante (Luchini et al., 1993; Donkin e Armentano, 1995).

Durante toda a fase experimental nenhum animal apresentou sintomatologia compatível com quadros de intoxicação por gossipol. Em todas as necropsias realizadas também não foram observadas alterações macroscópicas sugestivas de quadros de intoxicação por gossipol. Outros trabalhos, no entanto, são necessários para avaliação das concentrações de gossipol no plasma para que se possam fazer recomendações seguras sobre seu uso para esta categoria animal. 
Tabela 8. Concentração sanguínea de glicose $(\mathrm{mg} / \mathrm{dl})$ de bezerros alimentados com feno e concentrado (grupo-feno) ou alimento concentrado com caroço de algodão (grupo-algodão) antes do fornecimento dos alimentos (hora zero), três, seis e nove horas após a primeira alimentação do dia em diferentes idades

\begin{tabular}{cccccc}
\hline \multirow{2}{*}{$\begin{array}{c}\text { Idade } \\
\text { (dias) }\end{array}$} & Grupo & \multicolumn{4}{c}{ Horas após a alimentação } \\
\cline { 3 - 5 } & & 6 & 3 & 6 & 9 \\
\hline 45 & Feno & 67,8 & 66,5 & 64,2 & 66,2 \\
& Algodão & 68,2 & 62,3 & 66,2 & 64,3 \\
\hline \multirow{2}{*}{60} & Feno & 69,6 & 65,6 & 63,8 & 61,4 \\
& Algodão & 63,8 & 64,0 & 60,6 & 60,6 \\
\hline 75 & Feno & 62,5 & 59,5 & 58,3 & 60,5 \\
& Algodão & 59,0 & 58,5 & 58,0 & 63,0 \\
\hline \multirow{2}{*}{90} & Feno & 58,2 & 47,8 & 49,2 & 53,4 \\
& Algodão & 53,2 & 46,8 & 48,0 & 56,2 \\
\hline
\end{tabular}

CV: $12,1 \%$

\section{CONCLUSÕES}

A utilização do caroço de algodão em substituição ao feno de Tifton 85 foi efetiva como fonte de fibra na dieta, não provocou sintomatologia e morte condizentes com intoxicação por gossipol e não resultou em prejuízos no consumo de matéria seca e de água, no desenvolvimento ponderal dos animais, no perfil de fermentação ruminal e no desenvolvimento dos compartimentos do estômago.

\section{REFERÊNCIAS BIBLIOGRÁFICAS}

ANDERSON, M.J.; KHOYLOO, M.; WALTERS, J.L. Effect of feeding whole cottonseed on intake, body weight, and reticulorumen development of young Holstein calves. J. Dairy Sci., v.65, p.764-772, 1982.

BEHARKA, A.A.; NAGARAJA, T.G.; MORRIL, J.L. et al. Effects of form of the diet on anatomical, microbial, and fermentative development of the rumen of neonatal calves. $J$. Dairy Sci., v.81, p.1946-1955, 1998.

BERGMAN, E.N. Energy contributions of volatile fatty acids from the gastrointestinal tract in various species. Physiol. Ver., v.70, p.567590,1990

CHURCH, D.C. El ruminante - fisiologia digestiva y nutricion. Zaragoza: Acribia, 1993. $641 \mathrm{p}$.
COELHO, S.G. Ganho de peso e desenvolvimento do estômago de bezerros desaleitados aos trinta dias de idade $e$ alimentados com concentrado e com ou sem feno. 1999. 123f. Tese (Doutorado) - Escola de Veterinária, Universidade Federal de Minas Gerais, Belo Horizonte.

DELGADO, E.F. Caroço de algodão e milho grão, em diferentes formas físicas, na alimentação de vacas em lactação. 1994. 89f. Dissertação (Mestrado) - Escola Superior de Agricultura de Lavras, Universidade Federal de Lavras, Lavras.

DONKIN, S.S.; ARMENTANO, L.E. Insulin and glucagon regulation of gluconeogenesis in preruminating and ruminating bovine. J. Anim. Sci., v.73, p.546-551, 1995.

FRANCE, J.; SIDDONS, R.C. Volatile fatty acids production. In: FORBES, J. M.; FRANCE, J. (Eds). Quantitative aspects of ruminant digestion and metabolism. New York: Cab International, 1996. p.107-121.

FONTES, F.A.P.V.; COELHO, S.G.; LANA, A.M.Q., et. al. Desempenho de bezerros alimentados com dietas líquidas à base de leite integral ou soro de leite. Arq. Bras. Med. Vet. Zootec., v.58, p.212-219, 2006.

LUCHINI, N.D.; LANE, S.F.; COMBS, D.K. Preweaning intake and postweaning dietary energy effects on intake and metabolism of calves weaned at 26 days of age. J. Dairy Sci., v.76, p.255-266, 1993. 


\section{Bernardes et al.}

NOCEK, J.E.; HEALD, C.W.; POLAN, C.E. Influence of ration physical form and nitrogen availability on ruminal morphology of growing bull calves. J. Dairy Sci., v.67, p.334-343, 1984.

NUTRIENT requirements of dairy cattle. 6ed. Washington DC: National Academy of Sciences, 1989.

OFFICIAL methods of analysis. 16ed. Washington DC: AOAC, 1980.

ORTOLANI, E.L. Considerações técnicas sobre o uso da sonda esofágica na colheita do suco de rúmen de bovinos para mensuração do $\mathrm{pH}$. Arq. Esc. Vet. UFMG., v.33, p.269-275, 1981.

PLAZA, J.; MERINO, E.F.N.; RODRIGUEZ, M.E. et al. The effect of the level of ground hay in starter concentrates on calf performance. Cuban J. Agric. Sci., v.24, p.291-297, 1990.

QUIGLEY, J.D. III; CALDWELL, L.A.; SINKS, G.D. et al. Changes in plasma volatile fatty acids in response to weaning and feed intake in young calves. J. Dairy Sci., v.74, p.258-263, 1991 b.

RUSSEL, J.B. Effects of additives on in vitro ruminal fermentation: a comparison of monensin and bactracin, another gram-positive antibiotic. J. Anim. Sci., v.66, p.552-558, 1988.

SILVA, D. J. Análises de alimentos: métodos químicos e biológicos. Viçosa: Imprensa Universitária, 1981. 166p.

USER'S guide: statistics. Versão 6.12. Cary, NC: SAS Institute, 1995.

VAN SOEST, P.J.; ROBERTSON, J.B.; LEWIS, B.A. Methods for dietary fiber, neutral detergent fiber and non starch polysacharides in relation to animal nutrition. J. Dairy Sci., v.74, p.35833597, 1991.

VAZQUEZ-ANON, M.; HEIRICHS, A.J.; ALDRICH, J. M. Postweaning age effects on rumen fermentation end-products and digesta kinetics in calves weaned at 5 weeks of age. $J$. Dairy Sci., v.76, p.2742-2748, 1993.

VIRTALA, A.M.K.; MECHOR, G.D.; GROHN, Y.T. et al. The effect of calfhood diseases on growth of female dairy calves during the first three months of life in New York State. J. Dairy Sci., v.79, p.1040-1049, 1996. 